\title{
A New Distance Measure Based on Pythagorean Hesitant Fuzzy Sets and Its Application to Multicriteria Decision Making
}

\author{
Yanru Zhong ${ }^{\mathrm{a}}$, Xiuyan Guo ${ }^{\mathrm{a}}$, Hong Gao ${ }^{\mathrm{a}}$, Meifa Huang*b ${ }^{* \mathrm{~b}}$, Yuchu Qinc, and Xiaonan Luo ${ }^{\mathrm{a}}$ \\ ${ }^{a}$ Guangxi Key Laboratory of Intelligent Processing of Computer Images and Graphic, Guilin \\ University of Electronic Technology, Guilin, PR China; ${ }^{\mathrm{b}}$ School of Mechanical and Electrical \\ Engineering, Guilin University of Electronic Technology, Guilin, PR China; ${ }^{\mathrm{c}}$ School of Computing \\ and Engineering, University of Huddersfield, Huddersfield, United Kingdom
}

\begin{abstract}
The Pythagorean fuzzy set is characterized by five parameters, namely membership degree, non-membership degree, indeterminacy degree, strength of commitment about membership, and direction of commitment. The distance measure is important in solving the multicriteria decision-making problem with Pythagorean hesitant fuzzy (PHF) information. However, the existing distance measure considers the difference between the member ship degrees, the non-membership degrees, and the degrees of indeterminacy, but ignores the influence of the difference between the directions of PHF sets (PHFSs). The existing distance measure method may lead to unreasonable results sometimes. Inspired by above, the five parameters of PFS are extended to Pythagorean hesitant fuzzy set (PHFS) fully in this paper, generating new distance measures of PHFS and introducing some properties and theorems firstly. Then, the proposed method is applied in MCDM by considering the distance between the positive ideal solution and each alternative. Finally, to validate the effectiveness of the proposed method, a pragmatic experiment is introduced for comparisons with existing methods.
\end{abstract}

Keywords: Pythagorean hesitant fuzzy sets, Distance Measure, Multicriteria Decision Making, MCDM

\section{INTRODUCTION}

For Multicriteria decision-making (MCDM) problem ${ }^{[1]}$, Atanassov ${ }^{[2]}$ introduced a series of concepts of intuitionistic fuzzy set (IFS). Afterwards, Yager ${ }^{[4]}$ proposed the concept of Pythagorean fuzzy set (PFS) characterized by the membership degree and the non-membership degree of its elements. With respect to the original concept of PFSs, the membership degree and the non-membership degree are single values. When the evaluation information in MCDM may be not a single value, but several values simultaneously. Inspired by this, Torra ${ }^{[19]}$ proposed hesitant fuzzy sets (HFSs), which endorses the membership degree of an element to be a set of some feasible values between 0 and 1 . Considering the hesitant situation, $\mathrm{Xu}$ and Liang ${ }^{[14]}$ introduced HFSs into PFSs. The existing classical sets, including FSs, IFSs, HFSs and PFSs, can be regarded as special cases of $\mathrm{HPFSs}^{[14]}$.

Distance measure is an important topic in the fuzzy set theory and has been applied in $\mathrm{MCDM}^{[12]}$. It has attracted some researchers' interest and has been investigated further. A lot of distance measures of fuzzy sets and IFSs, PFSs, HFSs have been proposed. The most widely used distance measures for fuzzy sets are the Hamming distance, the Euclidean distance, and the Hausdorff metric ${ }^{[9,11-12]}$. Zhang and $\mathrm{Xu}^{[10]}$ proposed a distance measure of PFNs, which considers the differences of the membership degrees, non-membership degrees, and indeterminacy degrees. Li and Zeng ${ }^{[12]}$ proposed a series of new distance measures by considering the four fundamental parameters of PFN. Furthermore, Li and Zeng ${ }^{[13]}$ pointed out that distance measures necessitate considering five fundamental parameters of PFS and proposed a variety of distance measures for PFSs and PFNs. Xu and Liang ${ }^{[14]}$ proposed a distance measure of PHF numbers (PHFNs), which only considers the differences of the membership degrees, non- membership degrees, and indeterminacy degrees, but ignore the influence of the direction of PHF number (PHFN), which is a vital element of PHFN. To overcome such demerit, we propose a series of new distance measures by considering the five fundamental parameters of PHFN fully in this paper. Simultaneously, we propose the Distance formulas of PHFS by extending from the basic theories based on Refs ${ }^{[13,14]}$. Then, some properties and theorems of these formulas are proved. Finally, a numerical example is provided to illustrate the validity and applicability of the presented distance measures in MCDM under PHF environment.

* Corresponding author. Meifa Huang. E-mail: meifahuang@yeah.net (MH). 
The rest of this work is organized as follows. Section II gives a brief introduction to some related concepts. Section III explains the details of the proposed distance measures and theorem of PHFS, including the normalized Hamming distance, Euclidean distance, generalized distance. Section IV elaborates the proposed MCDM method via utilizing the proposed distance measures. A practical example, a set of experiments, and some comparisons are demonstrated in Section V. Section VI terminates the paper with a conclusion.

\section{PRELIMINARIES}

\subsection{Pythagorean Fuzzy Set}

Definition 1. ${ }^{[6,7]}$ Let $X$ be a universe of discourse. A PFS P in $X$ is given by

$$
P=\left\{<x, \mu_{p}(x), v_{p}(x)>\mid x \in X\right\},
$$

where $\mu_{p}: X \rightarrow[0,1]$ denotes the degree of membership and $v_{p}: X \rightarrow[0,1]$ denotes the degree of non-membership of the element $x \in \mathrm{X}$ to the set $\mathrm{P}$ with the condition that $0 \leq\left(\mu_{p}(x)\right)^{2}+\left(v_{p}(\mathrm{x})\right)^{2} \leq 1$. The degree of indeterminacy is given

by $\pi_{p}(x)=\sqrt{1-\left(\left(\mu_{p}(x)\right)^{2}+\left(v_{p}(\mathrm{x})\right)^{2}\right)}$. For convenience, Zhang and $\mathrm{Xu}^{[10]}$ called $\left(\mu_{p}(x), v_{p}(\mathrm{x})\right)$ as a PFN and is denoted by $p=\left(\mu_{p}, v_{p}\right)$.

Yager $^{[7,8]}$ gave another way to represent PFN, $p=\left(r_{p}, d_{p}\right)$, where $r_{p}$ is called the strength of $p$ and $d_{p}$ is called the direction of the strength $r_{p} . r_{p}$ and $d_{p}$ are associated with a pair of membership degree $\mu_{p}$ and non-membership degree $v_{p}$ indicating, respectively, the support for membership of $x$ in $\mathrm{P}$ and the support against membership of $x$ in P. The larger $r_{p}$ the stronger the commitment, the less the uncertainty. $d_{p}$ is essentially indicating on a scale of 0 to 1 how fully the strength $r_{p}$ is pointing to membership. If $d_{p}=1$, the direction of $r_{p}$ is completely to membership whereas $d_{p}=0$ the direction of the strength is completely to non-membership. The relationship between $p=\left(\mu_{p}, v_{p}\right)$ and $p=\left(r_{p}, d_{p}\right)$ is that $\mu_{p}=$ $r_{p} \cos \left(\theta_{p}\right), v_{p}=r_{p} \sin \left(\theta_{p}\right)$, where $d_{p}=1-\frac{2 \theta_{p}}{\pi}$. Besides, it's easily to obtain that $\theta_{p}=\operatorname{acrtan}\left(\frac{v_{p}}{\mu_{p}}\right)$ or $\theta_{p}=$ $\operatorname{acrsin}\left(\frac{v_{p}}{r_{p}}\right)$.

Known by the above analysis, Li and Zeng ${ }^{[13]}$ pointed out that each PFN $p$ is characterized by five parameters, membership degree $\mu_{p}$, non-membership degree $v_{p}$, indeterminacy or hesitancy degree $\pi_{p}$, strength $r_{p}$, and direction $d_{p}$.

Definition 2. ${ }^{[13]}$ Let $p_{1}, p_{2}$ be two PFNs, the normalized generalized distance between $p_{1}$ and $p_{2}$ is defined as follow:

$$
D_{G}\left(p_{1}, p_{2}\right)=\left[\frac{1}{5}\left(\left(\mu_{p_{1}}-\mu_{p_{2}}\right)^{\lambda}+\left(v_{p_{1}}-v_{p_{2}}\right)^{\lambda}+\left(\pi_{p_{1}}-\pi_{p_{2}}\right)^{\lambda}+\left(r_{p_{1}}-r_{p_{2}}\right)^{\lambda}+\left(d_{p_{1}}-d_{p_{2}}\right)^{\lambda}\right)\right]^{\frac{1}{\lambda}},
$$

where $\lambda \geqslant 1$.

If $\lambda=1$, then the distance $D_{G}\left(p_{1}, p_{2}\right)$ is changed into the Hamming distance $D_{H}\left(p_{1}, p_{2}\right)$.

If $\lambda=2$, then the distance $D_{G}\left(p_{1}, p_{2}\right)$ is changed into the Euclidean distance $D_{E}\left(p_{1}, p_{2}\right)$.

\subsection{Pythagorean Hesitant Fuzzy Set}

PHFS indicates that the membership of an element can be a set of Pythagorean fuzzy numbers (PFNs) possibilities. Wei et $\mathrm{al}^{[5]}$, Harish Garg $^{[3]}$ and Xu and Liang ${ }^{[14]}$ introduced the concept of PHFS, which is described as follow:

Definition 3. ${ }^{[3]}$ Let $X$ be a universe of discourse. A PHFS $P$ on $X$ is given by

$$
P=\{<x, h(x), g(x)>\mid x \in X\},
$$

in which $h(x)$ and $g(x)$ denote the membership and non-membership degrees of the element $x \in X$ to the set $P$, respectively, with the conditions $0 \leq \gamma, \eta \leq 1$ and $0 \leq\left(\gamma^{+}\right)^{2}+\left(\eta^{+}\right)^{2} \leq 1$ where $\gamma \in h(x), \eta \in g(x), \gamma^{+} \in h^{+}(x)=$ $\mathrm{U}_{\gamma \in h(x)} \max \{\gamma\}$ and $\eta^{+} \in g^{+}(x)=\mathrm{U}_{\eta \in g(x)} \max \{\eta\}$ for all $x \in X$. This pair is called an HPFE denoted by $p=h_{p}=$ $(h, g)$ or $p=h_{p}=(\{h\},\{g\})$. For the degree of indeterminacy of $h_{p}$, Xu and Liang ${ }^{[14]}$ deduced it as follow:

$$
\pi=\sqrt{1-\left(\left(l_{h}\right)^{-1} \sum_{\gamma \in h}(\gamma)^{2}+\left(l_{g}\right)^{-1} \sum_{\eta \in g}(\eta)^{2}\right)} .
$$


$l_{h}$ and $l_{g}$ denote the numbers of $h$ and $g$ respectively.

Definition 4. ${ }^{[14]}$ Let $h_{p 1}=\left(h_{1}, g_{1}\right)$ and $h_{p 2}=\left(h_{2}, g_{2}\right)$ be any two PHFNs, then the score function $S\left(h_{p i}\right)$ and the accuracy function $A\left(h_{p i}\right)$ of $h_{p i}(i=1,2, \ldots, n)$ are defined as follow:

$$
S\left(h_{p i}\right)=\left(\frac{1}{l_{h i}}\right) \sum_{\gamma \in h i}(\gamma)^{2}-\left(\frac{1}{l_{g i}}\right) \sum_{n \in g i}(\eta)^{2}, A\left(h_{p i}\right)=\left(\frac{1}{l_{h i}}\right) \sum_{\gamma \in h i}(\gamma)^{2}+\left(\frac{1}{l_{g i}}\right) \sum_{\eta \in g i}(\eta)^{2} .
$$

Then, to compare the PHFNs, Harish Garg ${ }^{[3]}$ proposed the principles for PHFNs as follow:

(1) If $S\left(h_{p 1}\right)>S\left(h_{p 2}\right)$, then $h_{p 1}$ is superior to $h_{p 2}$, denoted by $h_{p 1}>h_{p 2}$;

(2) If $S\left(h_{p 1}\right)=S\left(h_{p 2}\right)$, then

(a) If $A\left(h_{p 1}\right)>A\left(h_{p 2}\right)$, then $h_{p 1}$ is superior to $h_{p 2}$, denoted by $h_{p 1}>h_{p 2}$;

(b) If $A\left(h_{p 1}\right)=A\left(h_{p 2}\right)$, it is accounted for that $h_{p 1}$ is equivalent $h_{p 2}$, i.e., $h_{p 1} \approx h_{p 2}$.

$\mathrm{Xu}$ and Liang ${ }^{[14]}$ proposed the generalize distance of HPFS as follow:

Definition 5. ${ }^{[14]}$ Let $h_{p 1}=\left(h_{1}, g_{1}\right)$, and $h_{p 2}=\left(h_{2}, g_{2}\right)$ be two normalized PHFNs, then the hesitant Pythagorean fuzzy generalize distance between $h_{p 1}$ and $h_{p 2}$ as follow:

$$
d_{G}\left(h_{p 1}, h_{p 2}\right)=\left(2^{-1}\left(\left(l_{h}\right)^{-1} \sum_{k=1}^{l_{h}}\left|\left(\gamma_{1}^{(k)}\right)^{2}-\left(\gamma_{2}^{(k)}\right)^{2}\right|^{\lambda}+\left(l_{g}\right)^{-1} \sum_{k=1}^{l_{g}}\left|\left(\eta_{1}^{(k)}\right)^{2}-\left(\eta_{2}^{(k)}\right)^{2}\right|^{\lambda}+\left|\left(\pi_{1}\right)^{2}-\left(\pi_{2}\right)^{2}\right|^{\lambda}\right)\right)^{(\lambda)^{-1}} .
$$

If $\lambda=1$, then the distance $d_{G}\left(h_{p 1}, h_{p 2}\right)$ is changed into the Hamming distance $d_{H}\left(h_{p 1}, h_{p 2}\right)$.

If $\lambda=2$, then the distance $d_{G}\left(h_{p 1}, h_{p 2}\right)$ is changed into the Euclidean distance $d_{E}\left(h_{p 1}, h_{p 2}\right)$.

It's worth noting that the above generalize distance doesn't take the influence of the difference between the directions of PHF numbers into account.

\section{DISTANCE MEASURES OF PHFSs}

Inspired by the Refs. ${ }^{[13,14]}$, the main feature of PHFN also can be characterized by five parameters: $\mu_{p}, v_{p}, \pi_{p}, r_{p}$, and $d_{p}$. Considering the impact of the five parameters, the new distance measures of PHFNs are proposed as follow:

Definition 6. Let $p_{1}, p_{2}$ be two PHFNs, the normalized Hamming distance between $p_{1}$ and $p_{2}$ is defined as follow:

$$
D_{H}\left(p_{1}, p_{2}\right)=\frac{1}{5}\left(\frac{1}{l_{h}} \sum_{k=1}^{l_{h}}\left|\left(\gamma_{1}^{(k)}\right)^{2}-\left(\gamma_{2}^{(k)}\right)^{2}\right|+\frac{1}{l_{g}} \sum_{k=1}^{l_{g}}\left|\left(\eta_{1}^{(k)}\right)^{2}-\left(\eta_{2}^{(k)}\right)^{2}\right|+\left|\left(\pi_{1}\right)^{2}-\left(\pi_{2}\right)^{2}\right|+\left|\left(r_{1}\right)^{2}-\left(r_{2}\right)^{2}\right|+\left|\left(d_{1}\right)^{2}-\left(d_{2}\right)^{2}\right|\right) \text {. }
$$

The normalized Euclidean distance between $p_{1}$ and $p_{2}$ is defined as follow:

$$
\begin{aligned}
& D_{E}\left(p_{1}, p_{2}\right) \\
& =\sqrt{\frac{1}{5}\left(\frac{1}{l_{h}} \sum_{k=1}^{l_{h}}\left(\left(\gamma_{1}^{(k)}\right)^{2}-\left(\gamma_{2}^{(k)}\right)^{2}\right)^{2}+\frac{1}{l_{g}} \sum_{k=1}^{l_{g}}\left(\left(\eta_{1}^{(k)}\right)^{2}-\left(\eta_{2}^{(k)}\right)^{2}\right)^{2}+\left(\left(\pi_{1}\right)^{2}-\left(\pi_{2}\right)^{2}\right)^{2}+\left(\left(r_{1}\right)^{2}-\left(r_{2}\right)^{2}\right)^{2}+\left(\left(d_{1}\right)^{2}-\left(d_{2}\right)^{2}\right)^{2}\right)} .
\end{aligned}
$$

The normalized generalized distance between $p_{1}$ and $p_{2}$ is defined as follow:

$$
\begin{aligned}
& D_{G}\left(p_{1}, p_{2}\right) \\
& =\left(\frac{1}{5}\left(\frac{1}{l_{h}} \sum_{k=1}^{l_{h}}\left|\left(\gamma_{1}^{(k)}\right)^{2}-\left(\gamma_{2}^{(k)}\right)^{2}\right|^{\lambda}+\frac{1}{l_{g}} \sum_{k=1}^{l_{g}}\left|\left(\eta_{1}^{(k)}\right)^{2}-\left(\eta_{2}^{(k)}\right)^{2}\right|^{\lambda}+\left|\left(\pi_{1}\right)^{2}-\left(\pi_{2}\right)^{2}\right|^{\lambda}+\left|\left(r_{1}\right)^{2}-\left(r_{2}\right)^{2}\right|^{\lambda}+\left|\left(d_{1}\right)^{2}-\left(d_{2}\right)^{2}\right|^{\lambda}\right)\right)^{\frac{1}{\lambda},}
\end{aligned}
$$

where

$$
\pi_{1}=\sqrt{1-\left(\left(l_{h_{1}}\right)^{-1} \sum_{\gamma \in h_{1}}(\gamma)^{2}+\left(l_{g_{1}}\right)^{-1} \sum_{\eta \in g_{1}}(\eta)^{2}\right)}, \pi_{2}=\sqrt{1-\left(\left(l_{h_{2}}\right)^{-1} \sum_{\gamma \in h_{2}}(\gamma)^{2}+\left(l_{g_{2}}\right)^{-1} \sum_{\eta \in g_{2}}(\eta)^{2}\right)}, r_{1}=\sqrt{\left(l_{h_{1}}\right)^{-1} \sum_{\gamma \in h_{1}}(\gamma)^{2}+\left(l_{g_{1}}\right)^{-1} \sum_{\eta \in g_{1}}(\eta)^{2}},
$$


$r_{2}=\sqrt{\left(l_{h_{2}}\right)^{-1} \sum_{\gamma \in h_{2}}(\gamma)^{2}+\left(l_{g_{2}}\right)^{-1} \sum_{\eta \in g_{2}}(\eta)^{2}}, \theta_{1}=\arctan \left(\frac{\left(l_{g_{1}}\right)^{-1} \sum_{\eta \in g_{1}}(\eta)^{2}}{\left(l_{h_{1}}\right)^{-1} \sum_{\gamma \in h_{1}}(\gamma)^{2}}\right), \theta_{2}=\arctan \left(\frac{\left(l_{g_{2}}\right)^{-1} \sum_{\eta \in g_{2}}(\eta)^{2}}{\left(l_{h_{2}}\right)^{-1} \sum_{\gamma \in h_{2}}(\gamma)^{2}}\right), d_{1}=1-\frac{2 \theta_{1}}{\pi}, d_{2}=1-\frac{2 \theta_{2}}{\pi}$,

and $\lambda$ is constant and $\lambda \geqslant 1$. In this case, the distance $D_{G}\left(p_{1}, p_{2}\right)$ is a generalized one. On the basis of the value of $\lambda$, we can deduce the relationship among $D_{H}\left(p_{1}, p_{2}\right), D_{E}\left(p_{1}, p_{2}\right)$ and $D_{G}\left(p_{1}, p_{2}\right)$. It's noted that the algorithm of the normalization of PHFNs is cited from ${ }^{[14]}$. A parameter $\zeta$ is utilized to control the degree of normalization.

Property 1. When $\lambda=1$, then the distance $D_{G}\left(p_{1}, p_{2}\right)$ is reduced into the Hamming distance $D_{H}\left(p_{1}, p_{2}\right)$.

Property 2. When $\lambda=2$, then the distance $D_{G}\left(p_{1}, p_{2}\right)$ is reduced into the Euclidean distance $D_{E}\left(p_{1}, p_{2}\right)$.

Theorem 1. Let $p_{1}=\left(h_{1}, g_{1}\right)$ and $p^{+}=(\{1\},\{0\})$ be two HPFNs, then the normalized generalized distance $D_{G}\left(p_{1}, p^{+}\right)$ is defined as

$$
D_{G}\left(p_{1}, p^{+}\right)=\left(\frac{1}{5}\left(\left(l_{h}\right)^{-1} \sum_{k=1}^{l_{h}}\left|\left(\gamma_{1}^{(k)}\right)^{2}-1\right|^{\lambda}+\left(l_{g}\right)^{-1} \sum_{k=1}^{l_{g}}\left(\eta_{1}^{(k)}\right)^{2 \lambda}+\left(\pi_{1}\right)^{2 \lambda}+\left|\left(r_{1}\right)^{2}-1\right|^{\lambda}+\left|\left(d_{1}\right)^{2}-1\right|^{\lambda}\right)\right)^{(\lambda)^{-1}}
$$

where $p^{\prime+}$ is the normalization outcome of $p^{+}$by the comparison of $p$ and $p^{+}$,

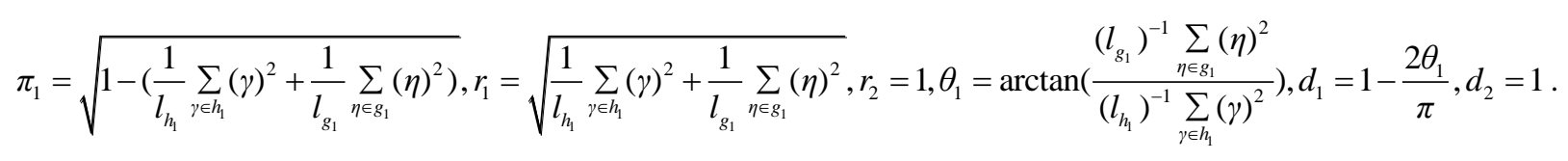

The proof of Theorem 1 is omitted here.

In Theorems 1, Equation (9) mainly relies on the five parameters: $\mu_{p}, v_{p}, \pi_{p}, r_{p}$, and $d_{p}$ of $p$ and $p^{+}$, which is needless to consider the order of the evaluation values of elements. Basic on Theorems 1, we further deduce the following property:

Property 3. When $\lambda=1, D_{G}\left(p_{1}, p^{\prime+}\right)$ is monotonic decreasing with the increase of $\gamma$. When $\lambda>1, D_{G}\left(p_{1}, p^{\prime+}\right)$ is monotonic increasing with the increase of $\gamma$. When $0<\lambda<1, D_{G}\left(p_{1}, p^{\prime+}\right)$ is monotonic increasing with the increase of $\gamma$.

\section{MCDM BASED ON DISTANCE MEASURE OF PHFS}

Let $X=\left\{x_{1}, x_{2}, \ldots, x_{m}\right\}$ be alternatives and $C=\left\{c_{1}, c_{2}, \ldots, c_{n}\right\}$ be attributes. Suppose that the weight vector of all attributes $W=\left\{w_{1}, w_{2}, \ldots, w_{n}\right\}^{T}$ is constant, which satisfies $0 \leqslant w_{j} \leqslant 1$ and $\sum_{j=1}^{n} w_{j}=1$. Under the PHF environment, the characteristics of the alternative $x_{i}(i=1,2, \ldots, m)$ in terms of the criteria in are expressed by the decision matrix $H=$ $\left(h_{i j}, g_{i j}\right)_{m \times n}$. For computing, we need to normalize $H$. Given the value of $\zeta$, we normalize the corresponding evaluation values of all alternatives in each criterion $c_{j}(j=1,2, \ldots, n)$ by employing Algorithm $1^{[14]}$, respectively.

For MCDM, we identify the positive ideal solution based on the normalized $H$. The positive ideal solution is denoted as $x^{+}=\left(v_{1}^{+}, v_{2}^{+}, \ldots, v_{n}^{+}\right)$. The positive ideal solution $x^{+}$is determined as

$$
x^{+}=\left(v_{1}^{+}, v_{2}^{+}, \ldots, v_{n}^{+}\right)=(p(\{1\},\{0\}), p(\{1\},\{0\}), \ldots, p(\{1\},\{0\}))
$$

Based on Theorem 1, the normalized generalized distance between the alternative $x_{i}$ and $x^{+}$is calculated as follow:

$$
\begin{aligned}
& D_{G}\left(x_{i}, x^{+}\right)=\sum_{j=1}^{n} w_{j} D_{G}\left(p\left(h_{i j}, g_{i j}\right), v_{j}^{+}\right)=\sum_{j=1}^{n} w_{j} D_{G}\left(p\left(h_{i j}, g_{i j}\right), p(\{1\},\{0\})\right) \\
& =\sum_{j=1}^{n} w_{j}\left(5^{-1}\left(\left(l_{h_{i j}}\right)^{-1} \sum_{\gamma \in h_{i j}}\left|(\gamma)^{2}-1\right|^{\lambda}+\left(l_{g_{i j}}\right)^{-1} \sum_{\eta \in g_{i j}} \eta^{2 \lambda}+\left(\pi_{i}\right)^{2 \lambda}+\left|\left(r_{i}\right)^{2}-1\right|^{\lambda}+\left|\left(d_{i}\right)^{2}-1\right|^{\lambda}\right)\right)^{(\lambda)^{-1}},
\end{aligned}
$$

where

$$
\pi_{i}=\sqrt{1-\left(\frac{1}{l_{h_{i j}}} \sum_{\gamma h_{i j}}(\gamma)^{2}+\frac{1}{l_{g_{i j}}} \sum_{\eta \in g_{i j}}(\eta)^{2}\right)}, r_{i}=\sqrt{\frac{1}{l_{h_{i j}}} \sum_{\gamma \in h_{i j}}(\gamma)^{2}+\frac{1}{l_{g_{i j}}} \sum_{\eta \in g_{i j}}(\eta)^{2}}, r^{+}=1, \theta_{i}=\arctan \left(\frac{\left(l_{g_{i j}}\right)^{-1} \sum_{\eta \in g_{i j}}(\eta)^{2}}{\left(l_{h_{i j}}\right)^{-1} \sum_{\gamma \in h_{i j}}(\gamma)^{2}}\right), d_{i}=1-\frac{2 \theta_{i}}{\pi}, d^{+}=1 .
$$


The following steps illustrate an approach in MCDM with the distance measures of PHFS felicitously.

Step 1. Construct the PHF decision matrix $H=p\left(h_{i j}, g_{i j}\right)_{m \times n}$. According to the practical decision-making problem, we determine alternatives $X=\left\{x_{1}, x_{2}, \ldots, x_{m}\right\}$ and the attributes $C=\left\{c_{1}, c_{2}, \ldots, c_{n}\right\}$. Meanwhile, we ascertain the weight vector of all attributes $W=\left\{w_{1}, w_{2}, \ldots, w_{n}\right\}^{T}, \lambda$ and $\zeta$, constructing the Pythagorean hesitant fuzzy decision matrix $H$.

Step 2. Normalize the decision matrix. Given the value of $\zeta$, we normalize the decision matrix $H$ by employing Algorithm 1 proposed in Ref. ${ }^{[14]}$. The result is showed at Table 2.

Step 3. Identify the positive solution. Based on Equation (10), the positive ideal solution $x^{+}=\left(v_{1}^{+}, v_{2}^{+}, \ldots, v_{n}^{+}\right)$is identified.

Step 4. Normalize the reference points. In the light of the normalized decision matrix, the reference points $x^{+}$is also normalized.

Step 5. Compute the generalized distance between the alternatives and the reference points. For each alternative $x_{i}$, the geometric distance $D_{G}\left(x_{i}, x^{+}\right)$between the alternative $x_{i}$ and $x^{+}$based on Equation (11) are further calculated.

Step 6. Rank the alternatives. According to the distance from the positive ideal solution, the ranking of alternatives is obtained. And then the shortest one is received.

\section{EXAMPLE, EXPERIMENT AND COMPARISONS}

\subsection{Example}

There are four attributes in Energy development strategy ${ }^{[14]}$ to be considered: (1) $C_{1}$ : economic; (2) $C_{2}$ : technological; (3) $C_{3}$ : environmental; and (4) $C_{4}$ : sociopolitical, i.e., $C=\left\{c_{1}, c_{2}, c_{3}, c_{4}\right\}$. Accordingly, the weight vector of the criteria is given $W=\{0.15,0.3,0.2,0.35\}^{T}$. In the energy project, it's assumed that there are five alternatives $X=\left\{x_{1}, x_{2}, x_{3}, x_{4}, x_{5}\right\}$. In PHF environment, some experts are invited to evaluate these alternatives with PHFNs. we elaborate the decision-making process of our proposed method for the energy project selection as follows:

Step 1. The Pythagorean hesitant fuzzy decision matrix $H=p\left(h_{i j}, g_{i j}\right)_{m \times n}$ is constructed which is shown at Table 1 .

Step 2. Based on the decision matrix $H$, the normalize the decision matrix $H^{\prime}$ (Suppose that $\zeta=0.5$ ) is shown in Table 2 .

Step 3\&4. According to the Table 2, the normalization of the reference points $x^{+}$can be determined as

$$
x^{+}=\left(v_{1}^{+}, v_{2}^{+}, v_{3}^{+}, v_{4}^{+}\right)=(p\{(1,1,1,1),(0,0,0)\}, p\{(1,1,1,1),(0,0,0)\}, p\{(1,1,1,1),(0,0,0)\}, p\{(1,1,1,1),(0,0,0)\}) .
$$

Step 5. By employing Equation (11), geometric distance $D_{G}\left(x_{i}, x^{+}\right)$between the alternative $x_{i}$ and $x^{+}$are further calculated. The smaller the geometric distance, the better the alternative. The calculated results are shown in Table 3.

Step 6. Based on Table 3, we can obtain the ranking of alternatives as follow: $x_{5}>x_{3}>x_{4}>x_{1}>x_{2}$.

Table 1. Pythagorean hesitant fuzzy decision matrix $H$.

\begin{tabular}{ccc}
\hline Alternatives & $\boldsymbol{C}_{\mathbf{1}}$ & $\boldsymbol{C}_{\mathbf{2}}$ \\
\hline$x_{1}$ & $p\{(0.3,0.4,0.5),(0.7,0.8)\}$ & $p\{(0.1,0.7,0.8,0.9),(0.2,0.4)\}$ \\
$x_{2}$ & $p\{(0.3,0.5),(0.6,0.7,0.8)\}$ & $p\{(0.2,0.5,0.6,0.7),(0.6,0.7)\}$ \\
$x_{3}$ & $p\{(0.6,0.7),(0.4,0.5,0.6)\}$ & $p\{(0.6,0.9),(0.2,0.3,0.4)\}$ \\
$x_{4}$ & $p\{(0.3,0.4,0.6,0.7),(0.5,0.7)\}$ & $p\{(0.2,0.4,0.7),(0.4,0.5,0.6)\}$ \\
$x_{5}$ & $p\{(0.1,0.3,0.6),(0.4,0.6)\}$ & $p\{(0.4,0.6,0.7,0.8),(0.1,0.2,0.3)\}$ \\
\hline Alternatives & $\boldsymbol{C}_{\mathbf{3}}$ & $p\{0.3,0.5,0.6,0.9,(0.2,0.3,0.4)\}$ \\
\hline$x_{1}$ & $p\{(0.2,0.4,0.5),(0.2,0.6,0.8)\}$ & $p\{(0.3,0.4,0.7),(0.4,0.5)\}$ \\
$x_{2}$ & $p\{(0.1,0.5,0.6,0.8),(0.2,0.5)\}$ & $p\{(0.4,0.6),(0.3,0.4,0.6)\}$ \\
$x_{3}$ & $p\{(0.3,0.5,0.7),(0.4,0.5,0.6)\}$ & $p\{(0.6,0.8,0.9),(0.2,0.3)\}$ \\
$x_{4}$ & $p\{(0.1,0.8),(0.3,0.4)\}$ & $p\{(0.3,0.6,0.7,0.9),(0.3,0.4)\}$ \\
$x_{5}$ & $p\{(0.7,0.8,0.9),(0.1,0.2,0.4)\}$ & \\
\hline
\end{tabular}


Table 2. The normalized decision matrix $H^{\prime}: \zeta=0.5$.

\begin{tabular}{ccc}
\hline Alternatives & $\boldsymbol{C}_{\mathbf{1}}$ & $\boldsymbol{C}_{\mathbf{2}}$ \\
\hline$x_{1}$ & $\{(0.3,0.4,0.5,0.4),(0.7,0.8,0.75)\}$ & $\{(0.1,0.7,0.8,0.9),(0.2,0.4,0.3)\}$ \\
$x_{2}$ & $\{(0.3,0.50 .4,0.4),(0.6,0.7,0.8)\}$ & $\{(0.2,0.5,0.6,0.7),(0.6,0.7,0.65)\}$ \\
$x_{3}$ & $\{(0.6,0.7,0.65,0.65),(0.4,0.5,0.6)\}$ & $\{(0.6,0.9,0.75,0.75),(0.2,0.3,0.4)\}$ \\
$x_{4}$ & $\{(0.3,0.4,0.6,0.7),(0.5,0.7,0.6)\}$ & $\{(0.2,0.4,0.7,0.45),(0.4,0.5,0.6)\}$ \\
$x_{5}$ & $\{(0.1,0.3,0.6,0.35),(0.4,0.6,0.5)\}$ & $\{(0.4,0.6,0.7,0.8),(0.1,0.2,0.3)\}$ \\
\hline Alternatives & $\boldsymbol{C}_{\mathbf{3}}$ & $\boldsymbol{C}_{\mathbf{4}}$ \\
\hline$x_{1}$ & $\{(0.2,0.4,0.5,0.35),(0.2,0.6,0.8)\}$ & $\{0.3,0.5,0.6,0.9,(0.2,0.3,0.4)\}$ \\
$x_{2}$ & $\{(0.1,0.5,0.6,0.8),(0.2,0.5,0.35)\}$ & $\{(0.3,0.4,0.7,0.5),(0.4,0.5,0.45)\}$ \\
$x_{3}$ & $\{(0.3,0.5,0.7,0.5),(0.4,0.5,0.6)\}$ & $\{(0.4,0.6,0.5,0.5),(0.3,0.4,0.6)\}$ \\
$x_{4}$ & $\{(0.1,0.8),(0.3,0.4)\}$ & $\{(0.6,0.8,0.9),(0.2,0.3)\}$ \\
$x_{5}$ & $\{(0.7,0.8,0.9,0.8),(0.1,0.2,0.4)\}$ & $\{(0.3,0.6,0.7,0.9),(0.3,0.4,0.35)\}$ \\
\hline
\end{tabular}

Table 3. The calculated results of geometric distance for each alternative: $\zeta=0.5$ and $\lambda=2$

\begin{tabular}{cccccc}
\hline The geometric distance & $x_{1}$ & $x_{2}$ & $x_{3}$ & $x_{4}$ & $x_{5}$ \\
\hline$D_{G}\left(x_{i}, x^{+}\right)$ & 0.5158 & 0.5769 & 0.4726 & 0.4874 & 0.4341 \\
Ranking & 4 & 5 & 2 & 3 & 1 \\
\hline
\end{tabular}

\subsection{Experiment}

The $H^{\prime}=p\left(h_{i j}, g_{i j}\right)_{m \times n}, \zeta, \lambda$ and $w$ are used as the inputs of distance measure of PHFS. First, $\zeta=0.5, \lambda=3$ for the geometric distance between the alternative $x_{i}$ and $x^{+}$. The results of the proposed distance measurements are shown in Table 4, which indicate that the ranking order of the proposed method is: $x_{5}>x_{3}>x_{4}>x_{1}>x_{2}$.

Table 4. The results of experiment 1.

\begin{tabular}{ccc}
\hline & Distance for each alternative & Ranking of alternatives \\
\hline$D_{H}\left(x_{i}, x^{+}\right)$ & $x_{1}=0.4587, x_{2}=0.5304, x_{3}=0.4404$, & $x_{5}>x_{3}>x_{4}>x_{1}>x_{2}$ \\
& $x_{4}=0.4441, x_{5}=0.3843$ & \\
$D_{E}\left(x_{i}, x^{+}\right)$ & $x_{1}=0.5158, x_{2}=0.5769, x_{3}=0.4726$, & \\
& $x_{4}=0.4874, x_{5}=0.4341$ & $x_{5}>x_{3}>x_{4}>x_{1}>x_{2}$ \\
$D_{G}\left(x_{i}, x^{+}\right)$ & $x_{1}=0.5625, x_{2}=0.6148, x_{3}=0.4976$, & \\
& $x_{4}=0.5215, x_{5}=0.4707$ & $x_{5}>x_{3}>x_{4}>x_{1}>x_{2}$ \\
\hline
\end{tabular}

To illustrate the impact of $\zeta$ and $\lambda$ to the energy selection, here are some analysis as follows:

(1) We discuss the impact of $\zeta$ to the selection. We normalize the decision matrix $\mathrm{H}$ and compute the distance of the alternatives with the different values of $\zeta$ under the value of $\lambda$. The comparison results are shown in Fig. 1.

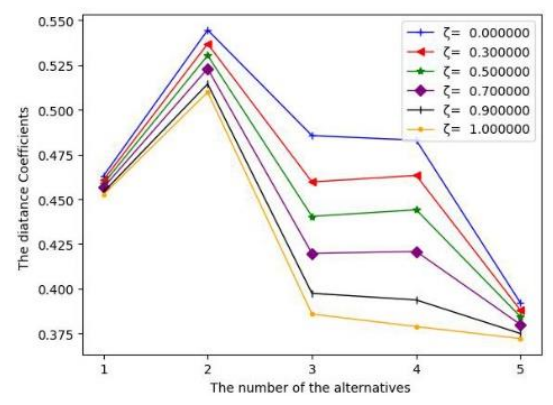

(1) $\lambda=1$

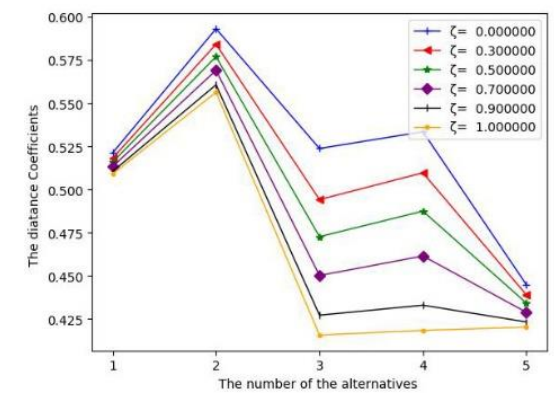

(2) $\lambda=2$

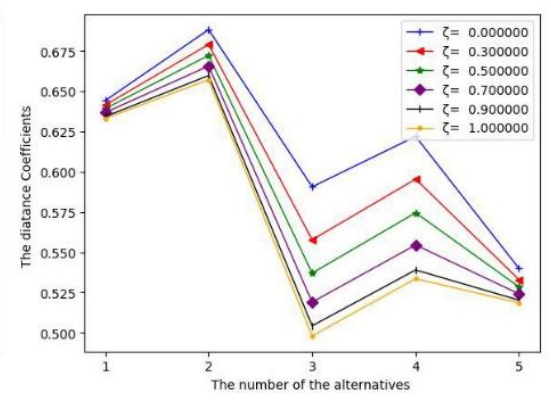

(3) $\lambda=5$ 
Figure 1. Comparison of the distance factor of the alternative with the different values of $\zeta$.

From Fig. 1, we discuss three cases of $\lambda$, i.e., $\lambda=1, \lambda=2$ and $\lambda=5$. The distance of each alternative with respect to the positive ideal solution $x^{+}$is decreasing with the increase of $\zeta$. Mostly, the ranking order $x_{5}>x_{3}>x_{4}>x_{1}>x_{2}$ holds.

(2) We discuss the impact of $\lambda$ to the selection. We normalize the decision matrix $\mathrm{H}$ and compute the distance of the alternatives with the different values of $\lambda$ under the value of $\zeta$. The comparison results are shown in Fig. 2. From Fig. 2, we discuss three cases of $\zeta$, i.e., $\zeta=0, \zeta=0.5$ and $\zeta=1.0$. The distance of each alternative with respect to the positive ideal solution $x^{+}$is increasing with the increase of $\lambda$. Mostly, alternative $x_{5}$ and alternative $x_{3}$ are comparable. When $\lambda \leq 3$, the ranking order is $x_{5}>x_{3}>x_{4}>x_{1}>x_{2}$. When $\lambda>3$, the ranking order $x_{3}>x_{5}>x_{4}>x_{1}>x_{2}$ holds.

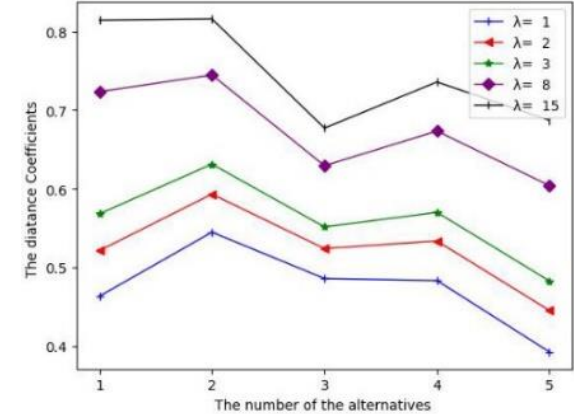

(1) $\zeta=0.0$

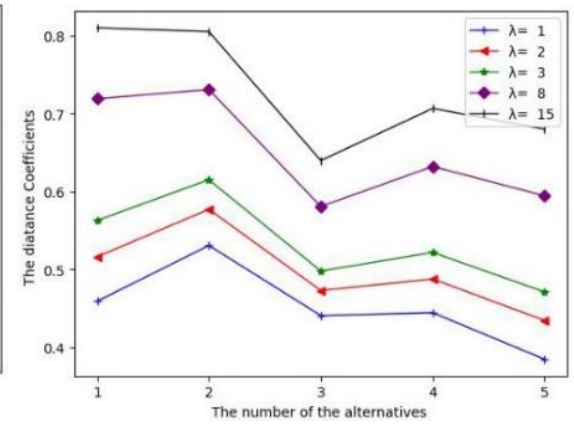

(2) $\zeta=0.5$

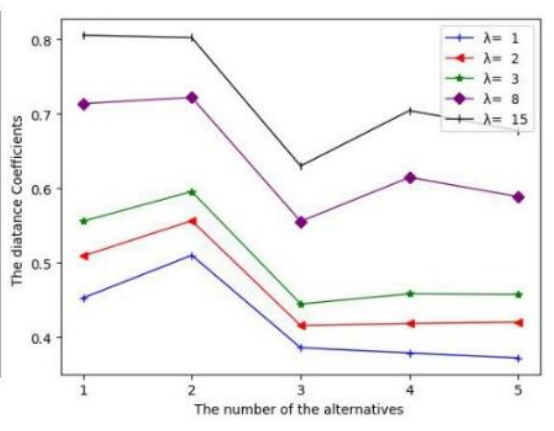

(3) $\zeta=1.0$

Figure 2. Comparison of the distance factor of the alternative with the different values of $\zeta$.

\subsection{Comparisons}

In this paper, we extend the distance measures of PHF to the difference between the directions of PHFNs based on the existing method ${ }^{[14]}$. In this section, we compare the methods of Refs. ${ }^{[3,14]}$ with our proposed method. For Ref. ${ }^{[14]}$, we calculate the geometric distance of each alternative with respect to the positive ideal solution $x^{+}$. Here, we suppose that $\zeta$ $=0.5$ and $\lambda=3$. We have reproduced the HPFWA operator and HPFWG operator, whose ranking is judged based on the score value of Definition 4, in Ref. ${ }^{[3]}$. The comparison results are shown in Table 5.

In Table 5, the decision results of the methods of Refs ${ }^{[3,14]}$ are consistent with our proposed method. It implies that our proposed method is efficacious. The advantages of our proposed method are summarized as follows: (1) Our proposed method is scalable to meet a variety of situations by adjusting its own parameters, i.e., it has very good flexibility and extension. (2) Our proposed method utilizes the satisfaction level of the alternative to the ideal solutions to make the decision. (3) Although the methods proposed in Refs. ${ }^{[3,14]}$ can generate the best option, they only consider the three possibilities of PHF, namely, $\mu_{p}, v_{p}$ and $\pi_{p}$. Therefore, the method we propose is more comprehensive.

Table 5. The results of comparisons.

\begin{tabular}{|c|c|c|c|c|c|c|}
\hline The method & $d_{G}\left(x_{1}, x^{+}\right)$ & $d_{G}\left(x_{2}, x^{+}\right)$ & $d_{G}\left(x_{3}, x^{+}\right)$ & $d_{G}\left(x_{4}, x^{+}\right)$ & $d_{G}\left(x_{5}, x^{+}\right)$ & Rank \\
\hline PHFWG & - & - & - & - & - & $x_{5}>x_{3}>x_{4}>x_{1}>x_{2}$ \\
\hline PHFWA & - & - & - & - & - & $x_{5}>x_{4}>x_{3}>x_{1}>x_{2}$ \\
\hline Xu's method [14] & 0.6539 & 0.6759 & 0.5658 & 0.5956 & 0.5624 & $x_{5}>x_{3}>x_{4}>x_{1}>x_{2}$ \\
\hline Our method & 0.5625 & 0.6148 & 0.4976 & 0.5215 & 0.4707 & $x_{5}>x_{3}>x_{4}>x_{1}>x_{2}$ \\
\hline
\end{tabular}

\section{CONCLUSION}

Distance measure is an important tool for distinguishing the objects in PHF environment. In this paper, we extent the concept of PFS to PHFS for a series of distance measures of PHFS which consider the five fundamental parameters fully. Simultaneously, by extending from the basic theories, the distance formulas of PHFS is introduced. Then, some properties and theorems of these formulas are proved. All the distances proposed enrich the theories of existing distances. Finally, a 
numerical example is provided to illustrate the validity and applicability of the presented distance measures, laying the foundation for future research of decision analysis.

\section{ACKNOWLEDGMENT}

This research was funded by the National Natural Science Foundation of China (No.61562016; No.61772149; No.61762028), Guangxi Key Laboratory of Images and Graphics Intelligent Processing (No. GIIP1805), and the Innovation Key Project of Guangxi Province (No. AA18118039-2).

\section{REFERENCES}

[1] Figueira, José, Greco S , Ehrgott M . Multiple Criteria Decision Analysis: State of the Art Surveys[M]. Springer New York, 2005.

[2] Atanassov K T . Two theorems for intuitionistic fuzzy sets[J]. Fuzzy Sets and Systems, 2000, 110(2):267-269.

[3] Garg H. Hesitant Pythagorean fuzzy sets and their aggregation operators in multiple attribute decision-making[J]. International Journal for Uncertainty Quantification, 2018, 8(3).

[4] Yager R R . Pythagorean fuzzy subsets[C]// IFSA World Congress and NAFIPS Annual Meeting (IFSA/NAFIPS), 2013 Joint. IEEE, 2013.

[5] Wu S J, Wei G W . Pythagorean hesitant fuzzy Hamacher aggregation operators and their application to multiple attribute decision making [J]. International Journal of Intelligent Systems, 2018, 97(3):24-39.

[6] Yager R R . Pythagorean fuzzy subsets[C]// IFSA World Congress and NAFIPS Annual Meeting (IFSA/NAFIPS), 2013 Joint. IEEE, 2013.

[7] Yager, Ronald R . Pythagorean Membership Grades in Multicriteria Decision Making[J]. IEEE Transactions on Fuzzy Systems, 2014, 22(4):958-965.

[8] Yager R R , Abbasov A M . Pythagorean Membership Grades, Complex Numbers, and Decision Making[J]. International Journal of Intelligent Systems, 2013, 28(5):436-452.

[9] Grzegorzewski P. Distance between intuitionistic fuzzy sets and/or interval-valued fuzzy sets based on the Hausdorff metric. Fuzzy Sets Syst 2004;148:319-328.

[10]Zhang X , Xu Z . Extension of TOPSIS to Multiple Criteria Decision Making with Pythagorean Fuzzy Sets[J]. International Journal of Intelligent Systems, 2014, 29(12).

[11] Dubois D, Prade H. Fuzzy sets and systems: Theory and applications. New York: AcademicPress; 1980.

[12] Li D , Zeng W . Distance Measure of Pythagorean Fuzzy Sets[J]. International Journal of Intelligent Systems, 2017.

[13]Zeng W, Li D, Yin Q. Distance and similarity measures of Pythagorean fuzzy sets and their applications to multiple criteria group decision making[J]. International Journal of Intelligent Systems, 2018, 33(11): 2236-2254.

[14] Liang D , Xu Z . The new extension of TOPSIS method for multiple criteria decision making with hesitant Pythagorean fuzzy sets[J]. Applied Soft Computing, 2017, 60:167-179.

[15] Atanassov, K. "Geometrical interpretation of the elements of the intuitionistic fuzzy objects." Preprint IMMFAIS-1-89, Sofia (1989).

[16] Atanassov, K.A second type of intuitionistic fuzzy sets. BUSEFAL 56, 66-70 (1993).

[17] Vassilev, P., R. Parvathi, and K. Atanassov. "Note on intuitionistic fuzzy sets of p-th type." Issues in Intuitionistic Fuzzy Sets and Generalized Nets 6 (2008): 43-50.

[18] Atanassov, Krassimir T., and Peter Vassilev. "On the intuitionistic fuzzy sets of n-th type." Advances in Data Analysis with Computational Intelligence Methods. Springer, Cham, 2018. 265-274.

[19] V. Torra, Hesitant fuzzy sets, Int. J. Intel. Syst. 25 (2010) 529-539. 